\title{
Severing Scale Structure from the Adjective*
}

\author{
E. Matthew Husband \\ University of South Carolina \\ husbande@mailbox.sc.edu
}

The scale structure of adjectives, whether an adjective measures on an open or closed scale, has certain grammatical consequences and is traditionally captured by encoding scale structure into an adjective's lexical representation and projecting it into the grammar. However, adjectives can exhibit scalar variability, suggesting that scale structure is not projected from the lexicon, but instead is constructed by the adjective's structural environment. I review a recent analysis of adjectival scale structure, observing that an economy condition with a single pos morpheme requires polysemy for these variable-behavior adjectives, and propose that splitting the pos morpheme and severing scale structure from the adjective captures scalar variability within a unified lexical entry without economy.

\section{Introduction}

Within a Kennedy-style analysis of adjectives, in which gradable adjectives denote measure functions, type $\langle e, d\rangle$, gradable adjectives must combine with some kind of degree morphology to derive a property of individuals, type $\langle e, t\rangle$ (Kennedy 1997). Although adjectives in their positive form lack overt degree morphology, it is argued that they combine with a covert pos morpheme, a piece of degree morphology which introduces a standard of comparison, $\operatorname{stnd}(g)$, and a relation between the measured degree of the individual and the standard, $\boldsymbol{R}$, as given in (1). Determining the relation and the standard for the pos morpheme depends on the structure of the scale measured by the adjective.

$$
\llbracket p o s \rrbracket=\lambda g \lambda x[\boldsymbol{R}(g(x))(\operatorname{stnd}(g))]
$$

Scale Structure The scales underlying adjectival measurement are defined by an open or closed set of degrees, an ordering, and a dimension of measurement, $\langle D, O, \Delta\rangle$. The structure of an adjective's scale is thought to affect the adjective's distribution and interpretation. Proportional modifiers like completely are acceptable with closed scale adjectives, while those like very are acceptable with open scale adjectives (Kennedy \& McNally 2005).

a. completely/??very straight/quiet/unknown/pure/safe/...

b. ??completely/very bent/loud/famous/impure/dangerous/...

Scale structure also affects the standard of comparison of an adjective in the positive form (Kennedy \& McNally 2005). Relative (i.e. open scale) adjectives have a contextually determined standard, as in (3). Absolute (i.e. closed scale) adjectives do not, requiring either a minimum (non-zero degree) standard, as in (4a), or a maximum (maximum degree) standard, as in (4b).

(3) a. The man is tall. b. The basketball player is tall.

(4) a. The rod is bent. b. The rod is straight.

* I would like to thank Marcin Morzycki for his guidance and helpful comments and suggestions while navigating the treacherous waters of adjectival meaning. 
Scale structure further affects entailments in comparatives and negation (Kennedy \& McNally 2005). Relative adjectives do not entail their antonyms in either comparatives, as in (5a), or negation, as in (6a). Absolute adjectives entail their antonyms in comparatives, as in (5b) and (5c), and negation, as in (6b) and (6c).

a. This door is larger than the window (but neither are large).

b. The rod is more bent than the stick, (\#but neither is bent).

c. This glass is more full than that one (\#but both are full).

a. This door is not large (but it's not small either).

b. The rod is not bent (\#though there is a small bend in the middle).

c. This glass is full (\#but it could be fuller).

Finally, scale structure interacts with the Sorities Paradox (Kennedy 2007). Relative adjectives give rise to the Sorities Paradox. Although the premise, P, and induction step, I, seem true, the conclusion, $\mathrm{C}$, is judged to be false, as seen in (7). Absolute adjectives do not give rise to the Sorities Paradox. The conclusion, $\mathrm{C}$, does not go through since the induction step, I, for both minimum and maximum standard adjectives is judged to be false, as seen in (8) and (9).

(7) P: A man who is 7 feet tall is tall.

$\mathrm{I}$ Any man who is 1 inch shorter than a tall man is tall. $\mathrm{T}$

C: Therefore, any man who is 3 feet tall is tall. $\quad \mathrm{F}$

P: A rod that has 10 degrees of bend is bent

I: Any rod that is 1 degree less bent than a bent rod is bent. $\quad \mathrm{F}$

$\mathrm{C}$ : Therefore, any rod that has 0 degrees of bend is bent. ?

P: A theater in which every seat is occupied is full. $T$

I: Any theater with one fewer occupied seat than a full theater is full. $\quad \mathrm{F}$

C: Therefore, any theater in which half of the seats are occupied is full. ?

A Case Study of Dry The adjective $d r y$ exhibits scalar variability. As a permanent stable property, dry has a relative interpretation. As a transient property, dry has an absolute interpretation Kennedy \& McNally (2005: 370). Proportional modifiers, for instance, diagnose open and closed scale $d r y$, as seen in (10a) and (10b).

a. This region of the country is ??completely/very dry.

b. The glasses are completely/??very dry.

Relative (i.e. open scale) and absolute (i.e. closed scale) dry also have different standards of comparison. Relative dry has a contextually determined standard, as in (11a). Absolute dry has a maximum standard, as in (11b).
a. This region of the country is dry.
b. The glasses are dry.

Relative and absolute dry generate different entailments in comparatives and negation. Relative $d r y$ does not entail non-dryness, as in (12a) and (13a). Absolute dry entails non-dryness, as in (12b) and (13b).

(12) a. This region of the country is drier than that one (though both are dry).

b. The glasses are drier than the plates (\#though both are dry).

a. This region of the country is not dry (but it's not wet either).

b. The glasses are not dry (\#though they're not wet either). 
Finally, relative and absolute $d r y$ interact differently with the Sorities Paradox. For relative $d r y$, the conclusion, $\mathrm{C}$, is judged to be false, although the premise, $\mathrm{P}$, and induction step, I, seem true, as seen in (14). For absolute $d r y$, the induction step, I, is judged to be false, thus the conclusion, $\mathrm{C}$, does not go through, as seen in (15).

P: A region of the country with $35 \%$ humidity is dry.

I: Any region with $1 \%$ more humidity than a dry region is dry. $\mathrm{T}$

C: Therefore, any region of the country with $100 \%$ humidity is dry. F

P: A glass with $0 \%$ moisture on it is dry. $\quad T$

I: Any glass with $1 \%$ more moisture on it than a dry glass is dry. F

C: Therefore, any glass with $50 \%$ moisture on it is dry.

\section{Kennedy (2007)}

Kennedy (2007) provides a unified meanings for the pos morpheme, proposing that it introduces a standard in which the individual "stands out" relative to the scale. The function stand-out is given in (17) (Kennedy 2004).

$$
\begin{aligned}
& \llbracket p o s \rrbracket=\lambda g \lambda x[\text { stand-out }(g(x))] \\
& \text { stand-out }(g(x))= \begin{cases}g(x)=\max (g) & \text { if } \max (g) \text { is defined, } \\
g(x) \succ \min (g) & \text { if } \min (g) \text { is defined, } \\
g(x) \succeq \operatorname{stnd}(g) & \text { elsewhere. }\end{cases}
\end{aligned}
$$

To ensure that adjectives with closed scales are linked to the minimum/maximum point of their scale, Kennedy (2007) proposes a principle of interpretative economy.

Interpretative Economy: Maximize the contribution of the conventional meanings of the elements of a sentence to the computation of its truth conditions.

Under this analysis, adjectives exhibiting scalar variability must be polysemous since Interpretative Economy requires the stand-out standard to be based on the scale structure of the adjective. If $d r y$ measures a closed scale, stand-out and Interpretative Economy derive only the absolutive interpretation. If $d r y$ measures an open scale, stand-out and Interpretative Economy derive only the relative interpretation. Thus $d r y$ must come in two flavors, one absolute and one relative.

$$
\begin{aligned}
\llbracket[\text { pos } \operatorname{dry}] \rrbracket & =\lambda x[\operatorname{stand}-\operatorname{out}(\operatorname{dry}(x))] & \\
& =\lambda x[\operatorname{dry}(x)=\mathbf{m a x}(\operatorname{dry}(x))] & \left(\text { dry as closed scale, } D_{[0, \infty)}\right) \\
& =\lambda x[\operatorname{dry}(x) \succeq \operatorname{stnd}(\operatorname{dry}(x))] & \left(\text { dry as open scale, } D_{(0, \infty)}\right)
\end{aligned}
$$

\section{Severing Scale Structure}

A Kennedy-style analysis of scalar variability leaves us with a question: Can we unify the lexical entries for adjectives which exhibit variable scalar behaviors? An initial clue lies in the definition of the standout function. A closer look at this function reveals that it actually encodes three possibilities for pos morphemes. I propose splitting Kennedy's (2007) pos morpheme into three functional items, one for each type of closure. pos $_{\text {open }}$ in (20a) introduces a contextually determined standard, creating an open scale environment. pos lower-closed in (20b) introduces a minimum absolute standard which picks out the minimum degree of a scale and creates a lower closed scale environment. pos upper-closed $_{\text {in }}$ (20c) introduces a maximum absolute standard which picks out the maximum degree of a 
scale and creates an upper closed scale environment.

$$
\begin{aligned}
& \text { a. } \llbracket p o s_{\text {open }} \rrbracket=\lambda g \lambda x[g(x) \succeq \operatorname{stnd}(g)] \\
& \text { b. } \llbracket p o s_{\text {lower-closed }} \rrbracket=\lambda g \lambda x[g(x) \succ \min (g)] \\
& \text { c. } \llbracket p o s_{\text {upper-closed } \rrbracket}=\lambda g \lambda x[g(x)=\mathbf{m a x}(g)]
\end{aligned}
$$

Note that spliting Kennedy's pos morpheme only removes the need for Interpretative Economy. Since the adjective still projects its scale structure, the scale structure imposed by pos morpheme must be redundant with that of the adjective. To remove this redundancy, I propose that the pos morpheme alone structures the scale of the adjective. While adjectives are still measure functions which map an individual to a degree on a scale, they do not project the content of that scale into the grammar. As such, traditionally "closed" or "open" scale adjectives have no grammatical requirement that they be interpreted at the maximum/minimum degree or above a standard degree. To the extent any adjective has some restriction, it is because of the scalar concept that underlies what that adjective measures.

Consider the case of $d r y$ given the denotation in (21). [pos open $d r y]$ introduces stnd, selecting a relative standard. The scale underlying dry can coerce into this relative meaning since $D_{(0, \infty)}$ is a subset of $D_{[0, \infty)}$. [pos upper-closed $\left.d r y\right]$ introduces max, selecting a maximum absolute standard. The scale underlying $d r y$ also allows for this maximum absolute meaning since $\max \left(D_{[0, \infty)}\right)$ is defined at 0 . Finally, [pos lower-closed $\left.d r y\right]$ introduces min, selecting a minimum absolute standard. However, the scale underlying dry does not allow a minimum absolute meaning since $\min \left(D_{[0, \infty)}\right)$ is not defined. This conflict between the grammar's requirements and the scalar concept's flexibility results in unacceptability since no amount of contextual support can resolve the conflict, an analysis very much in the spirit of Borer's (2005) structuring sense theory.

$$
\begin{aligned}
& \llbracket \operatorname{dry} \rrbracket=\lambda x[\operatorname{dry}(x)] \text { where } d r y: f: M \subseteq U \rightarrow\left\langle D_{[0, \infty)}, \prec, \text { moisture }\right\rangle \\
& \text { a. } \llbracket\left[\text { pos }_{\text {open }} \operatorname{dry}\right] \rrbracket=\lambda x[\operatorname{dry}(x) \succeq \operatorname{stnd}(\operatorname{dry})] \quad \text { (relative dry) } \\
& \text { b. } \left.\quad\left[\operatorname{pos}_{\text {upper-closed }} \operatorname{dry}\right] \rrbracket=\lambda x[\operatorname{dry}(x)=\max (\operatorname{dry})] \quad \text { (max absolute } d r y\right) \\
& \text { c. } \llbracket\left[\operatorname{pos}_{\text {lower-closed }} \mathrm{dry}\right] \rrbracket=\lambda x[\operatorname{dry}(x) \succ \min (\text { dry })] \quad \text { (min absolute } d r y \text { ) }
\end{aligned}
$$

\section{Conclusions}

Severing scale structure from the adjective avoids economy and redundancy while maintaining a unified lexical entry. Adjectives are embedded in certain structural environments and, to the extent their concept fits the environment's scalar requirements, they are acceptable. This analysis supports constructionist approaches where structures and general lexical meanings together create more specialized meanings.

\section{References}

Borer, Hagit. 2005. Structuring sense. Oxford University Press.

Kennedy, Chris. 1997. Projection the adjective: The syntax and semantics of gradability and comparison. Ph.D. thesis, University of California, Santa Cruz.

Kennedy, Chris. 2004. Towards a grammar of vagueness. Draft of a paper given at the 2003 Princeton Semantics Workshop.

Kennedy, Chris. 2007. Vagueness and grammar: The semantics of relative and absolute gradable adjectives. Linguistics and Philosophy 30(1). 1-45.

Kennedy, Chris \& Louise McNally. 2005. Scale structure, degree modification, and the semantics of gradable predicates. Language 81. 345-381. 